\title{
OBESIDADE, SÍNDROME METABÓLICA E IMPACTO NA SAÚDE DO IDOSO BRASILEIRO
}

\author{
Daniel Sarmento Bezerra|* \\ Camila Irineu Moura Alencar Falcão II \\ Rayza Prado Barreto Santos Ramiro III
}

Felipe Brandão dos Santos Oliveira IV

\section{RESUMO}

Os idosos são a parcela da população brasileira que mais cresceu na última década e o envelhecimento está intimamente associado ao aumento na prevalência de doenças crônicas não transmissíveis, a exemplo das doenças endócrinas e cardiovasculares. Estas doenças impactam na longevidade e na qualidade de vida ao se desdobrarem em danos físicos, psicológicos e sociais aos idosos. O presente trabalho consiste em uma revisão integrativa de literatura, nos bancos de dados da Biblioteca Virtual em Saúde (BVS): LILACS (Literatura Latino Americana e do Caribe em Ciências da Saúde), utilizando os descritores: obesidade, síndrome metabólica e idoso, e teve como objetivo discutir sobre os impactos que a obesidade e a síndrome metabólica promovem ao idoso brasileiro. Os resultados mostram que os idosos da zona urbana apresentam menos chances de adoecimento que os de zona rural uma vez que são mais informados e possuem maior acesso aos serviços de saúde. Ademais, existe uma tendência da população mundial em agregar hábitos saudáveis, atividades físicas e práticas alternativas de higiene mental, o que corrobora com o aumento da qualidade de vida e diminuição das principais doenças crônicas. Ao mesmo tempo, a análise do IMC - Índice de Massa Corporal, em larga escala, pode ser um instrumento epidemiológico de grande valor para predizer agravos e antecipar políticas adequadas a cada região do país, aproveitando o fato da mulher ser mais propensa ao autocuidado.

PALAVRAS-CHAVE: Obesidade. Serviços de Saúde para Idosos. Saúde Pública.

Estudante de Medicina, Faculdade de Medicina Nova Esperança e Departamento de Medicinal ORCID: 0000-0003-1430-4796

Autor correspondente: sarmentomeddaniel@gmail.com Estudante de Medicina, Faculdade de Medicina Nova Esperança e Departamento de Medicina. " ORCID: 0000-0003-4732-2747 Estudante de Medicina, Faculdade de Medicina Nova Esperança e Departamento de Medicina. ${ }^{\text {II }}$ ORCID: 0000-0003-1070-1946 Faculdade de Medicina Nova Esperança - Departamento de Medicina. IV ORCID: 0000-0002-1682-4755 


\section{INTRODUÇÃO}

Os idosos são a parcela da população brasileira que mais cresceu na última década e o envelhecimento acelerado está intimamente associado ao aumento na prevalência de doenças crônicas não transmissíveis, a exemplo das doenças endócrinas e cardiovasculares ${ }^{1}$. Um dos maiores problemas de saúde pública é a obesidade, que é o acúmulo excessivo de gordura corporal nas células do tecido adiposo do corpo humano, sendo este acúmulo diretamente relacionado aos hábitos alimentares ${ }^{2}$.

Segundo a ABESO (Associação Brasileira para Estudos de Obesidade e Síndrome Metabólica), 52,5\% dos brasileiros estavam obesos em 2014. Em um estudo paralelo efetuado pelo IBGE (Instituto Brasileiro de Geografia e Estatística) ficou demonstrado que entre 2003-2013 este valor saltou para a casa dos $60 \%$. Ao menos 82 milhões de brasileiros estão com sobrepeso, sendo que o sexo feminino representa $58,2 \%$. Ademais, o estudo alerta para fatores como idade, sexo, genética e hábitos de vida, já que os homens tendem a engordar a partir dos 25 anos e têm alimentação mais desregrada. Já as mulheres engordam aos 35 e possuem maior chance de síndrome metabólica por questões hormonais ${ }^{8}$.

Ao mesmo tempo que a população urbana de idosos cresce e toma para si a resolução dos problemas diários de forma cada vez mais empoderada, temos um grande desafio sendo enfrentado cotidianamente pelos profissionais de saúde que é alcançar a população idosa das pequenas cidades e das zonas rurais. Estes cidadãos muitas vezes convivem com a total ignorância acerca de conhecimentos simples e que podem evitar diversas doenças 3 .

Os hábitos de vida das populações idosas das diferentes regiões do país variam muito e traduzem ao pesquisador algumas possíveis ameaças a saúde quando colhidas informações sobre o IMC - Índice de Massa Corporal dos entrevistados. Este indicador é simples, rápido e possibilita ao médico inferir sobre diversas doenças que possam estar ameaçando a vida do paciente, sendo uma ferramenta bastante eficaz no combate as doenças endócrinas segundo a Organização Mundial da Saúde ${ }^{1,4}$.

O IMC é calculado dividindo-se o peso pela altura elevada ao quadrado e o normal é que esteja entre 18,5 e 24,9; sendo sobrepeso até 30 e obesidade quando ultrapassar $30^{1}$. Por sua vez, pacientes que têm IMC maior que 40 já se encontram dentro dos candidatos a procedimentos cirúrgicos para redução de peso. $O$ Brasil é um país de proporções continentais e possui, por sua vez, cerca de 19 milhões de pessoas com IMC maior ou igual a 30, o que caracteriza obesidade tipo $1^{4}$.

As sociedades de cardiologia de todo o mundo alertam para os riscos da obesidade como fator de risco para uma série de doenças que, unidas, provocam a síndrome metabólica, principalmente em idosos, quando o metabolismo é mais lento. Os idosos obesos são mais propensos a desenvolver e/ou agravar doenças de base como: hipertensão, diabetes tipo 2, doenças cardiovasculares, entre tantas doenças endocrinometabólica. Por fim, conviver com as várias comorbidades causadas pela síndrome metabólica ${ }^{1,3}$.

A síndrome metabólica - SM é um fenômeno endócrino que agrega hipertensão arterial, dislipidemia e obesidade abdominal e muitos estudos indicam que as pessoas são afetadas em razão direta com a idade. Ademais, a presença da SM em paciente idoso aumenta em até cinco vezes os riscos cardiovasculares e cerebrovasculares ${ }^{1}$. Por outro lado, novos critérios, foram agregados para o melhor diagnóstico da SM: circunferência abdominal aumentada, hiperglicemia de jejum, HDL- co- 
lesterol baixo, hipertrigliceridemia e pressão arterial elevada, sendo que o paciente para ser diagnosticado com SM deve ter ao menos 3 dos supracitados ${ }^{1,5}$.

Desta forma, esta pesquisa, através da questão norteadora "quais os impactos que

\section{MATERIAL E MÉTODOS}

O presente estudo tratou-se de uma pesquisa de revisão integrativa de literatura. Esse método tem por finalidade agrupar e sintetizar resultados de pesquisas acerca de um dado tema, de uma forma organizada, a fim de contribuir para um aprofundamento no conhecimento sobre a questão abordada 6 .

Realizou-se uma revisão sobre as produções cientificas com base nos impactos causados pela síndrome metabólica como também da obesidade na saúde do idoso brasileiro, no contexto nacional, na área de saúde. Esse método permite "[...] a busca, a avaliação crítica e a síntese das evidências disponíveis do tema investigado"7 $\mathrm{O}$ uso deste método permite como resultado a atual situação de conhecimento sobre o tema abordado, a implementação de intervenções na assistência à saúde e a identificação de lacunas que necessitem de mais aprofundamento no assunto.

Para elaboração desse estudo, foram utilizadas as seis etapas da revisão integrativa citadas $^{6}$, com o intuito de organizar as informações coletadas.

$1^{\mathrm{a}}$ etapa: elaboração da questão norteadora. Sua definição é a fase mais importante, pois determina a identificação do que deve ser abordado para contemplar o tema proposto.

$2^{a}$ etapa: busca ou amostragem na literatura. A busca foi realizada de forma ampla e diversificada nas bases de dados confiáveis, sendo levado em consideração que a deter- a obesidade e a síndrome metabólica têm na saúde do idoso brasileiro?", teve como objetivo buscar e discutir artigos que relacionem a obesidade, a síndrome metabólica e as consequentes implicações e impactos na saúde do idoso brasileiro. minação dos critérios de inclusão e exclusão para seleção do material deveria ser realizada em concordância com a pergunta norteadora.

$3^{\text {a }}$ etapa: coleta dos dados. Nesta etapa ficou definido o que seria extraído dos estudos selecionados através do uso de um instrumento previamente elaborado, com a finalidade de organizar as informações-chave de maneira concisa para construção do estudo.

$4^{a}$ etapa: análise crítica dos estudos incluídos. Nesta fase foi realizada a análise dos dados de forma detalhada para garantir a validade da revisão. Sempre levando em consideração a questão norteadora como base para toda análise.

$5^{\text {a }}$ etapa: discussão dos resultados. Nesta fase foram discutidos os resultados obtidos na pesquisa e realizou-se uma análise crítica acerca do que foi evidenciado.

$6^{\text {a }}$ etapa: apresentação da revisão integrativa. Esta é a fase onde o estudo foi devidamente elaborado. Contendo informações suficientes para que o leitor faça uma análise sobre o estudo realizado.

Os artigos foram selecionados na base de dados da Biblioteca Virtual em Saúde (BVS): LILACS (Literatura Latino Americana e do Caribe em Ciências da Saúde). Os descritores utilizados foram: obesidade, síndrome metabólica e idoso, através dos operadores booleanos "AND" e "OR", com o objetivo de facilitar as buscas aos materiais indexados nas bases de 
dados.

O universo da pesquisa foi composto por artigos online no campo da saúde, relacionados com a saúde do idoso. Para isso foram determinados os seguintes critérios de inclusão: estarem disponíveis em texto completo nas bases de dados selecionadas, serem publicações do tipo artigo, no período de 2012 a 2017, no idioma português. Os critérios de exclusão foram: dissertações e teses, e não contemplarem o tema proposto.

As informações contidas no referido instrumento visaram a caracterização e as contribuições das publicações selecionadas, no sentido de atender aos objetivos propos- tos para a investigação. São elas: identificação do artigo, base de dados, ano e origem da publicação, método e periódico.

$\mathrm{Na}$ busca inicial com a utilização dos descritores e critérios de inclusão, foram localizados 9.577 artigos sendo 5.160 disponíveis na íntegra. Após a utilização dos filtros e selecionados 116 artigos, destes apenas 20 possuíam dados compatíveis com a revisão. Após retirar artigos repetidos, 07 compuseram a dissertação. Os dados dos estudos incluídos na pesquisa foram categorizados, analisados e discutidos estabelecendo-se relações com a fundamentação teórica em foco.

\section{RESULTADOS E DICUSSÃO}

QUADRO 1 - Distruibuição dos artigos que elaboraram a amostra segundo título, periódico, objetivo e resultado.

\begin{tabular}{|c|c|c|c|c|}
\hline & TÍTULO & PERIÓDICO & OBJETIVO & RESULTADO \\
\hline E1 & $\begin{array}{l}\text { Correlação entre indica- } \\
\text { dores de obesidade ab- } \\
\text { dominal e lipídeos séricos } \\
\text { em idosos. }\end{array}$ & Rev. Assoc. Med. Bras. & $\begin{array}{l}\text { Verificar a correlação en- } \\
\text { tre os indicadores antro- } \\
\text { pométricos de obesidade } \\
\text { abdominal e lipídeos séri- } \\
\text { cos em idosos. }\end{array}$ & $\begin{array}{l}\text { Foram avaliados } 321 \text { ido- } \\
\text { sos (67,6\% mulheres). Ve- } \\
\text { rificaram-se correlações } \\
\text { significativas entre os in- } \\
\text { dicadores de obesidade } \\
\text { abdominal e as frações } \\
\text { lipídicas apenas no grupo } \\
\text { das mulheres. A circunfe- } \\
\text { rência de cintura foi pre- } \\
\text { ditora de 9,2\% da variação } \\
\text { do HDL e a relação circun- } \\
\text { ferência e quadril de } 7,3 \% \\
\text { da variação do TG. }\end{array}$ \\
\hline E2 & $\begin{array}{l}\text { Associação da obesidade } \\
\text { com a percepção de saú- } \\
\text { de negativa em idosas: } \\
\text { um estudo em bairros de } \\
\text { baixa renda de Curitiba, } \\
\text { Sul do Brasil. }\end{array}$ & Rev. Salud Pública & $\begin{array}{l}\text { Analisar a associação en- } \\
\text { tre o estado nutricional } \\
\text { (sobrepeso e obesidade) } \\
\text { e a percepção de saúde } \\
\text { em idosas de regiões de } \\
\text { baixa renda do município } \\
\text { de Curitiba, Brasil. }\end{array}$ & $\begin{array}{l}\text { Idosas obesas tiveram } \\
\text { duas vezes mais chances } \\
\text { de ter uma percepção ne- } \\
\text { gativa de saúde. }\end{array}$ \\
\hline
\end{tabular}




\begin{tabular}{|c|c|c|c|c|}
\hline E3 & $\begin{array}{l}\text { Potencializando um gru- } \\
\text { po de terceira idade de } \\
\text { uma comunidade rural. }\end{array}$ & Rev Esc Enferm USP & $\begin{array}{l}\text { Descrever as fases do } \\
\text { processo de potenciali- } \\
\text { zação de um grupo de } \\
\text { terceira idade de uma co- } \\
\text { munidade rural. }\end{array}$ & $\begin{array}{l}\text { O grupo foi capaz de aco- } \\
\text { lher a novidade, expres- } \\
\text { sar angústias e compar- } \\
\text { tilhar alegrias. Os novos } \\
\text { conhecimentos levaram } \\
\text { a mudanças com adapta- } \\
\text { ção a hábitos saudáveis } \\
\text { e melhora no relaciona- } \\
\text { mento interpessoal. }\end{array}$ \\
\hline E4 & $\begin{array}{l}\text { Prevalência de Síndrome } \\
\text { Metabólica em Idosos e } \\
\text { Concordância entre Qua- } \\
\text { tro Critérios Diagnósticos. }\end{array}$ & Arq. Bras. Cardiol & $\begin{array}{l}\text { Determinar a prevalência } \\
\text { de SM em idosos por qua- } \\
\text { tro critérios diagnósticos } \\
\text { e a concordância entre } \\
\text { esses. }\end{array}$ & $\begin{array}{l}\text { A prevalência de SM foi } \\
\text { elevada em todos os cri- } \\
\text { térios utilizados no estu- } \\
\text { do. }\end{array}$ \\
\hline E5 & $\begin{array}{l}\text { Peso e altura autorrefe- } \\
\text { ridos para determinação } \\
\text { do estado nutricional de } \\
\text { adultos e idosos: validade } \\
\text { e implicações em análises } \\
\text { de dados. }\end{array}$ & Cad. Saúde Pública & $\begin{array}{l}\text { Avaliar a validade das } \\
\text { medidas de peso e altura } \\
\text { autorreferidas, e o efeito } \\
\text { dos erros sobre o IMC de } \\
\text { adultos e idosos. }\end{array}$ & $\begin{array}{l}\text { As avaliações de autoper- } \\
\text { cepção precisam ser bem } \\
\text { referenciadas e analisa- } \\
\text { das já que nem sempre os } \\
\text { dados são verdadeiros. } \\
\text { Dependendo do convívio } \\
\text { social pode haver omis- } \\
\text { são das informações. } \\
\text { Neste estudo os resulta- } \\
\text { dos se mostraram pouco } \\
\text { discrepantes. }\end{array}$ \\
\hline E6 & $\begin{array}{l}\text { Prevalência de Síndrome } \\
\text { metabólica em idosos de } \\
\text { comunidades urbana e } \\
\text { rural participantes do HI- } \\
\text { PERDIA do município de } \\
\text { Coimbra/MG, Brasil. }\end{array}$ & Invest. Educ. Enferm & $\begin{array}{l}\text { Identificar a prevalência } \\
\text { da síndrome metabólica } \\
\text { (MS) e a influência do } \\
\text { gênero e local de residên- } \\
\text { cia dos idosos atendidos } \\
\text { pela Estratégia de Saúde } \\
\text { da Família no município } \\
\text { de Coimbra (estado de } \\
\text { Minas Gerais, Brasil). }\end{array}$ & $\begin{array}{l}\text { As mulheres apresenta- } \\
\text { ram maior prevalência de } \\
\text { MS - Síndrome Metabóli- } \\
\text { ca (urbano }=40 \% \text {, rural = } \\
37 \% \text { que os homens (ur- } \\
\text { bano = } 13 \% \text {, rural = } 22 \% \text { ). } \\
\text { Sendo as mulheres mais } \\
\text { expostas à obesidade } \\
\text { (urbano = } 80,4 \% \text {, rural = } \\
78,6 \% \text { do que os homens, } \\
\text { independentemente do } \\
\text { local de residência. }\end{array}$ \\
\hline E7 & $\begin{array}{l}\text { Fatores associados à Sín- } \\
\text { drome Metabólica em } \\
\text { idosos: uma revisão inte- } \\
\text { grativa. }\end{array}$ & $\begin{array}{l}\text { Revista Kairós Geron- } \\
\text { tologia }\end{array}$ & $\begin{array}{l}\text { Identificar os fatores de } \\
\text { risco associados à Síndro- } \\
\text { me Metabólica em } \\
\text { idosos, por meio de uma } \\
\text { revisão integrativa, } \\
\text { no período de } 2003 \text { a } \\
2013 \text {. }\end{array}$ & $\begin{array}{l}\text { A prática regular de ati- } \\
\text { vidade física, associada a } \\
\text { uma dieta rica } \\
\text { em frutas, verduras, e ce- } \\
\text { reais integrais, influencia } \\
\text { no controle da síndrome. }\end{array}$ \\
\hline
\end{tabular}

O Brasil possui uma perspectiva de ter, até o ano de 2040, ao menos 52 milhões de idosos. Isto significa que eles serão $25 \%$ da população brasileira. O sistema de saúde pública não está qualificado nem adaptado para aten- der um aumento tão significativo no número de usuários e medidas político-administrativas precisam ser postas em prática com urgência para evitar o colapso total dos serviços médicos em um futuro próximo ${ }^{2,3}$. 
Avaliando-se pacientes brasileiros com média de 65 anos, pode-se concluir que ao menos $88 \%$ das mulheres eram portadoras de SM, e entre os homens, o índice chegou a 74\%. Uma das explicações para que mulheres tenham maior prevalência nos índices de SM, além de maus hábitos de vida, é que ao entrar na menopausa a ausência de estrogênio que por sua vez contribui para uma elevação do colesterol o que culmina em dislipidemias $1,2,5$.

Neste ínterim, as discussões acerca da SM são de grande importância já que esta síndrome endócrina é bastante recorrente entre os idosos, causa distúrbios cardiovasculares como tromboflebites, tromboembolismo, infarto agudo do miocárdio, trombose venosa profunda; além de eventos cerebrovasculares como tromboembolias e acidentes vasculares isquêmicos e hemorrágicos. Ainda, limitações que geram grandes custos orçamentários aos cofres públicos; ainda, muitas vezes levando o paciente a óbito ${ }^{1}$.

Sabendo que a SM é um agravo de alto impacto para a saúde do idoso brasileiro se faz urgente que haja uma força tarefa focada para melhorar os índices de sedentarismo e maus hábitos alimentares prevalentes neste grupo. Através de campanhas de educação e promoção de saúde, que são instrumentos fáceis e baratos de se realizar, com a finalidade de modificar o pensar e empoderar os idosos para uma vida mais plena e saudável ${ }^{1,4,5,6}$.

Os maus hábitos de vida provocam doenças cardíacas, renais e endócrinas; levando o idoso a uma rotina de viagens em busca de atendimento médico longe de suas regiões de origem. Estes deslocamentos são caros e desgastantes, inclusive, promovendo a piora e o adoecimento desse idoso. Diversos estudos ao redor do mundo evidenciam que os números da circunferência de homens e mulheres aumentam com a idade - o limite máximo é $94 \mathrm{~cm}$ para homens e $80 \mathrm{~cm}$ para mulheres propiciando um dos fatores de risco dos mais importantes para a instalação da $\mathrm{SM}^{1,2,4}$.

Ao mesmo tempo em que se discutem formas de melhorar os padrões de vida da população idosa, através de programas que incentivem melhores hábitos alimentares e redução do sedentarismo, temos um instrumento de grande valia no estudo epidemiológico da obesidade e de fácil aplicabilidade que é o programa de Vigilância dos Fatores de Risco e Proteção para Doenças Crônicas por Inquérito Telefônico (VIGITEL), que une dados de IMC acrescidos de dados socioculturais e de hábitos de vida 5 .

Serviços como o supracitado são importantes principalmente para que se atinja a população rural e seja possível desenhar estratégias especificas que possam ser implementadas por longos períodos em cada área descoberta. As barreiras que atravancam o acesso dos idosos aos serviços de saúde não são apenas geográficas; são econômicas, sociais, culturais e funcionais. Assim, neste grupo, a realização de trabalhos de ação social coletiva, através da educação em saúde, pode ocasionar uma consciência de aceitação e superação das limitações impostas pelas comorbidades que porventura venham a existir 3,7 .

Estas ações de educação em saúde devem ser realizadas através de práticas interativas e com o compartilhamento de experiาências vividas, demonstrando interesse e humanizando as práticas de saúde utilizadas. O engajamento social dos idosos entre si busca a solidariedade no combate não só a doenças crônicas como psíquicas. A junção de informações e hábitos saudáveis concorrem para que o idoso adoeça menos e não precise de atendimento médico-hospitalar ${ }^{3}$.

Ao compararmos o povo brasileiro em suas diversas regiões é importante discutir que as pesquisas com IMC são mais disseminadas que as que investigam a circunferência abdominal dos pacientes. Ainda que estas sejam mais predominantes em relação às que 
investigam perfis lipídicos de idosos, existe uma resposta muito diversa com conclusões extremas para riscos cardiovasculares, já que lugares mais distantes como a região Norte possuem hábitos alimentares e culturais bem distintos da região Sudeste. Esta mais industrializada que aquela ${ }^{1,4}$.

\title{
CONSIDERAÇÕES FINAIS
}

Ao compreender que o Brasil é um país com muitas desigualdades e de uma pluralidade impar em sua cultura e hábitos de vida, é possível começar a formar algumas ideias acerca de como prevenir doenças e agravos na população idosa. A partir de políticas públicas adequadas as diversas regiões é possível conscientizar esse idoso e fazer com que ele se previna contra diversas doenças que possam reduzir sua qualidade de vida. Doenças sérias como a Síndrome Metabólica implicam em outras comorbidades como hipertensão arterial de difícil controle, diabetes e doença aterosclerose que remetem ao uso contínuo não só de medicamentos como procedimentos e cirurgias, muitas vezes indisponíveis nas regiões mais distantes do país.

Regiões mais cosmopolitas possuem melhores condições médico-hospitalares para atendimento dos seus idosos ao contrário das regiões rurais mais afastadas dos grandes centros urbanos. Sendo assim, estudos epidemiológicos locais e regionais são imprescindíveis e precisam de uma manutenção ativa para que haja uma melhor abordagem dos planos de promoção de saúde em todo o território nacional. Ainda, o desbravamento dos sertões, por equipes de saúde, e a manutenção de campanhas de promoção e educação em saúde são ferramentas indispensáveis para que haja discernimento, independência e autonomia.

\section{OBESITY, METABOLIC SYNDROME AND IMPACT ON THE HEALTH OF THE ELDERLY BRAZILIAN: AN INTEGRATIVE REVIEW}

\begin{abstract}
The elderly Brazilian population that has grown the most in the last decade and aging is closely associated with the increase in the prevalence of chronic non-communicable diseases, such as endocrine and cardiovascular diseases. These diseases impact on longevity and quality of life as they unfold in physical, psychological and social damages to them. The present work consists of an integrative review of literature, in the databases of the Biblioteca Virtual em Saúde (BVS): LILACS (Latin American and Caribbean Literature in Health Sciences), using the descriptors: obesity, metabolic syndrome and elderly, and had the objective to discuss about the impacts that obesity and the metabolic syndrome promote to the Brazilian elderly. The results show that the elderly in the urban zone are less likely to become ill than rural people, since they are more informed and have greater access to health services. In addition, there is a tendency of the world population in aggregating healthy habits, physical activities and alternative practices of mental hygiene; which corroborates with the increase of the quality of life and decrease of the main
\end{abstract}


chronic diseases. At the same time, the analysis of BMI - body mass index, on a large scale, can be a valuable epidemiological tool to predict problems and anticipate policies appropriate to each region of the country, taking advantage of the fact that women are more prone to self-care.

KEYWORDS: Obesity. Health Services for the Elderly. Public health.

\section{REFERÊNCIAS}

1. ROCHA FL, MENEZES TN, MELO RLP, PEDRAZA DF. Correlação entre indicadores de obesidade abdominal e lipídeos séricos em idosos. Rev. Assoc. Med. Bras. 2013 Jan 59(1).

2. VAGETTI GC, BARBOSA-FILHO VC, MOREIRA NB, OLIVEIRA V, SCHIAVINI L, MAZZARDO O, CAMPOS W. Associação da obesidade com a percepção de saúde negativa em idosas: um estudo em bairros de baixa renda de Curitiba, Sul do Brasil. Rev. salud pública, 2012 Nov 14(6).

3. MACHADO ARM, SANTOS WS, DIAS FA, TAVARES DMS, MUNARI DB. Potencializando um grupo de terceira idade de uma comunidade rural. Rev Esc Enferm USP, 2015 49(1): p. 96-103.

4. CALIXTO SCS, VINAGRE RMFD, ROCHA GF, FRANÇA TG. Prevalência de Síndrome Metabólica em Idosos e Concordância entre Quatro Critérios Diagnósticos. Arq. Bras. Cardiol, 2014 Mar 102(3).

5. DUCA GFD, CHICA DAG, SANTOS JV, KNUTH
AG, CAMARGO MBJ, ARAÚJO CL. Peso e altura autorreferidos para determinação do estado nutricional de adultos e idosos: validade e implicações em análises de dados. Cad. Saúde Pública, 2012 jan 28(1).

6. PAULA JAT, MOREIRA OC, SILVA CD, SILVA DS, AMORIM PRS. Prevalência de Síndrome metabólica em idosos de comunidades urbana e rural participantes do HIPERDIA do município de Coimbra/MG, Brasil. Invest. educ. enferm, 2015 Mai 33(2).

7. NASCIMENTO JPS, MENEZES MAA, MALLMANN DG, JARDIM VCFS. Fatores associados à Síndrome Metabólica em idosos: uma revisão integrativa. Revista Kairós Gerontologia, 2015 Abr 18(2): p. 283-97.

8. Associação Brasileira de Estudos em Obesidade e Síndrome Metabólica. [homepage na internet]. Quase 60\% dos brasileiros estão acima do peso, revela IBGE. [acesso em 06 mar 2019]. Disponível em: http:www.abeso.gov.br. 